\title{
Efeito do substrato, do pericarpo e de tratamentos pré-germinativos na emergên- cia de plântulas de pata-de-elefante (Beaucarnea recurvata Lem.) ${ }^{(1)}$
}

\author{
YARA BRITO CHAIM JARDIM ROSA(2), OSMAR ROSA ESPÍRITO SANTO(3), RODRIGO CARVALHO GUIMARÃES ${ }^{(3)}$, \\ MARICHEL CANAZZA DE MACEDO(4), MARIANA BENTO TATARA(5) e EDGARD JARDIM ROSA JUNIOR(6)
}

\begin{abstract}
RESUMO
Devido seu aspecto escultural, a pata-de-elefante (Beaucarnea recurvata Lem. - Ruscaceae) é uma espécie de potencial econômico, necessitando de estudos que visem à maximização de sua produção. Este trabalho avaliou o efeito de diferentes substratos, da presença do pericarpo e de tratamentos pré-germinativos na emergência de plântulas em dois experimentos distintos. No primeiro experimento, sementes com e sem pericarpo, armazenadas por 120 dias em condições ambientais, foram semeadas em substratos constituídos por: 1- Latossolo Vermelho Distroférrico (LVD); 2- areia grossa (AG); 3- 1/3 de $\mathrm{LVD}+1 / 3$ de $\mathrm{AG}+1 / 3$ de moinha de carvão (MC). Utilizou-se o delineamento experimental inteiramente casualizados e os tratamentos foram arranjados em esquema fatorial $2 \times 3 \mathrm{com}$ quatro repetições de 25 sementes. Os maiores valores de porcentagem $(\% \mathrm{E})$ e índice de velocidade (IVE) de emergência foram obtidos da semeadura de sementes sem pericarpo e os maiores valores de IVE, \%E, Matéria Seca Total (MS) e Diâmetro do Caule (DC) foram observados nas plantas cultivadas no substrato composto por LVD+AG+ MC. No segundo experimento, sementes com pericarpo armazenadas por um ano em condições ambientais tiveram o pericarpo removido e receberam os seguintes tratamentos pré-germinativos: imersão em água natural por 12, 24, 36, 48, 72 e 96 horas e imersão em bioestimulante por 5 minutos nos volumes de 40, 80 e 120 $\mathrm{mL} \mathrm{kg}^{-1}$ de sementes. Foi utilizado o delineamento experimental inteiramente casualizado com quatro repetições de 25 sementes e a semeadura foi realizada em areia grossa. Não houve efeito dos tratamentos pré-germinativos na \%E sendo os maiores valores de IVE registrados quando se utilizou a embebição em água por 96 horas.
\end{abstract}

Palavras-chave: Beaucarnea recurvata, planta ornamental, germinação.

\begin{abstract}
Effect of substrate, pericarp and pre-germinative treatments on ponytail seedling emergence

On account of its structural appearance, the ponytail tree (Beaucarnea recurvata Lem. - Ruscaceae) is a specie of economic potential requiring studies that aim a maximization on its production. This work evaluated the effect of different substrates, pericarp and pregerminative treatments on seedling emergence in two distinct experiments. In the first experiment, seeds with or without pericarp and stored for 120 days at environmental conditions were sowed in substrates consisting of: 1Oxisol (Typic Haplustox) (LVD); 2- thick sand (AG); $3-1 / 3$ of LVD $+1 / 3$ of $A G+1 / 3$ of dust charcoal (MC). It was used a completely randomized experimental design and the treatments were arranged in a 2 x 3 factorial with four replicates of 25 seeds. The highest values of percentage $(\% \mathrm{E})$ and speed index (IVE) emergence were obtained from the sowing of seeds without pericarp and the \%E, IVE, total dry matter (MS) and stem diameter (DC) highest values were observed in the plants cultivated in substrate composed by LVD $+\mathrm{AG}+\mathrm{MC}$. In the second experiment, seeds with pericarp and stored for one year under environmental conditions had their pericarp removed and they had the followings pre-germinative treatments: immersion in natural water for 12, 24, 36, 48, 72 and 96 hours and in biostimulant for 5 minutes in the volumes of 40,80 and $120 \mathrm{~mL}$ seed kg-1. It was used the completely randomized design with four replications of 25 seeds and the sowing was realized in thick sand. It was not observed effect of pre-germinative treatments on $\% \mathrm{E}$ and the IVE highest values were observed when it was used seed immersion in water during 96 hours.
\end{abstract}

Keywords: Beaucarnea recurvata, ornamental plant, germination.

\section{INTRODUÇÃO}

O setor de flores e plantas ornamentais vem se destacando no agronegócio brasileiro, necessitando de informações técnicas relativas às várias etapas de produção e de comercialização (TERCEIRO NETO et al., 2004; TANIO e SIMÕES, 2005). Além disso, vale destacar que o país possui notórias vantagens para especializar-se na produção de flores, como o clima, a disponibilidade de terra, água, energia e mão-de-obra (MARQUES e CAIXETA FILHO, 2003).

As sementes constituem a via de multiplicação mais empregada na implantação de cultivos de plantas ornamentais. Nesse sentido, a busca de conhecimentos sobre as condições ótimas para a sua germinação e posterior emergência de plântulas é fundamental para a pesquisa científica e fornece informações valiosas sobre a propagação das espécies (VARELA et al., 2005).

\footnotetext{
(1) Trabalho recebido para publicação em 29/12/2010 e aprovado em 19/03/2014;

(2) Doutora emAgronomia, Professora Associada 3 da Universidade Federal da Grande Dourados (UFGD). Rodovia Dourados-Itahum, Km 12, CEP:79804970. Dourados - MS. yararosa@ufgd.edu.br;

(3) Alunos de Agronomia da Universidade Federal da Grande Dourados (UFGD). osmar_r_e_s@yahoo.com.br; rcg_83@hotmail.com;

${ }^{(4)}$ Bacharel em Ciências Biológicas, Mestre em Produção Vegetal, Doutoranda em Produção Vegetal do Programa de Pós-Graduação em Agronomia da

UFGD. marichelcanazza@zipmail.com.br;

${ }^{(5)}$ Bacharel em Ciências Biológicas, Mestre em Produção Vegetal, Técnica de laboratório da UFGD. marianabtatara@gmail.com.br;

${ }^{(6)}$ Doutor em Agronomia, Professor Associado 3 da UFGD. edgardjunior@ufgd.edu.br.
} 
A ocorrência de germinação heterogênea dentro de um mesmo lote de sementes distribui o estabelecimento de indivíduos ao longo do tempo e possibilita a sobrevivência da população às fases inadequadas ao seu desenvolvimento no campo (CARVALHO e NAKAGAWA, 2000). Para viveiristas e produtores, entretanto, essa característica é uma desvantagem, pois induz grande desuniformidade de tamanho entre as mudas e maior demanda de tempo na sua produção, além de maior risco de perda de sementes por deterioração, já que estas permanecem mais tempo no solo antes de germinarem.

Segundo Rosseto e Alves (2008), procedimentos que propiciem o maior número possível de plantas estabelecidas, ao mesmo tempo e a partir de sementes, são necessários. A remoção parcial ou total do pericarpo dos frutos pode aumentar a velocidade de emergência das plântulas maximizando a produção de mudas (PEREIRA et al., 1996). Da mesma forma, a aplicação exógena de reguladores vegetais pode resultar em ações similares (VIEIRA e CASTRO, 2002), resultando em maior percentual germinativo das sementes (GARCIA et al., 2006).

A imersão das sementes em água é outro método que, além da fácil execução, tem proporcionado resultados satisfatórios para várias espécies, tais como tucumã ( $A s$ trocaryum aculeatum Meyer) (FERREIRA e GENTIL, 2006) e tarumã (Vitex megapotamica (Spreng.) Moldenke) (VIANNA e KOEHLER, 2007).

O substrato também influencia na germinação das sementes e posterior desenvolvimento das plântulas, já que fatores como aeração, estrutura, capacidade de retenção de água, grau de infestação de patógenos, entre outros, podem variar de acordo com o tipo de material utilizado. Portanto, sua escolha deve ser criteriosa, pois influencia a qualidade das plantas e os custos de produção (POPINIGIS, 1985; GROLLI, 1991; FLIGLIOLIA et al., 1993; CARVALHO e NAKAGAWA, 2000).

A pata de elefante (Beaucarnea recurvata Lem.) é uma espécie semi-arbustiva, nativa do México, de três a cinco metros de altura, que apresenta tronco geralmente não ramificado, muito dilatado na base com um tufo de folhas coriáceas e côncavas na extremidade. Tal característica confere à espécie grande valor ornamental (LORENZI e SOUZA, 1999), podendo ser utilizada como planta envasada ou em paisagismo.

A espécie apresenta inflorescências eventuais, eretas, grandes, densas, com numerosas flores pequenas e esbranquiçadas, formadas no outono e de pouco valor ornamental e, embora propagada por meio de sementes (LORENZI e SOUZA, 1999), não há informações sobre os fatores que afetam a sua germinação.
A intensa utilização da pata de elefante na composição de jardins públicos e privados da região da Grande Dourados-MS, e a dependência do Estado em relação às regiões produtoras brasileiras, aliada à escassez de bibliografia técnica relativa à sua propagação, tornam evidente a necessidade de estudos que visem à maximização da produção de mudas dessa espécie.

Diante do exposto, este trabalho teve por objetivos avaliar o efeito de diferentes substratos, da presença do pericarpo e de tratamentos pré-germinativos na emergência de plântulas de B. recurvata.

\section{MATERIAL E MÉTODOS}

O trabalho foi conduzido na área de Jardinocultura da Faculdade de Ciências Agrárias (FCA) da Universidade Federal da Grande Dourados (UFGD) em Dourados-MS, de abril de 2008 a maio de 2009. A cidade de Dourados está localizada nas coordenadas 22 13'16"S e 54 $48^{\prime} 02^{\prime \prime} \mathrm{W}$ com altitude média de $452 \mathrm{~m}$. As médias anuais de temperatura do ar máxima e mínima são de 24 e $20{ }^{\circ} \mathrm{C}$, respectivamente, com precipitação total anual entre 1250 e $1500 \mathrm{~mm}$. O clima regional é do tipo Cwa mesotérmico úmido, segundo a classificação de KÖPPEN (1948).

Frutos maduros de pata-de-elefante (Beaucarnea recurvata Lem.) foram colhidos de cachos produzidos por duas plantas com aproximadamente 25 anos de idade, localizadas na área urbana de Dourados em abril de 2008. Os frutos foram secos à sombra por sete dias e após esse período foram acondicionados em recipientes plásticos desprovidos de tampa e armazenados sob condições ambientais $\left(23 \pm 2{ }^{\circ} \mathrm{C}\right)$ até a instalação dos seguintes experimentos:

2.1. Efeito dos substratos e do pericarpo na emergência de plântulas de pata de elefante:

Após 120 dias de armazenamento, foram contadas 600 sementes sendo que 300 delas tiveram o pericarpo removido manualmente e 300 não, constituindo dois lotes (com e sem pericarpo) que foram semeadas a um centímetro de profundidade, em vasos de polietileno branco, com capacidade de $180 \mathrm{~mL}$. Em cada vaso foi semeada uma semente e os vasos foram preenchidos com os seguintes substratos: 1- Latossolo Vermelho Distroférrico (LVD); 2- areia grossa (AG); 3- $1 / \frac{1}{3}$ de $L V D+1 / 3$ de $A G+$ $1 / 3$ de moinha de carvão (MC) -em volume- cujos atributos químicos e físicos, determinados conforme metodologia proposta pela EMBRAPA (1997), são apresentados na Tabela 1. 
Tabela 1. Valores dos atributos físicos e químicos dos substratos utilizados no cultivo de pata de elefante (Beaucarnea recurvata Lem.). Dourados, UFGD, 2009.

Table 1. Values of physic and chemistry attributes of substrata utilized in ponytail tree (Beaucarnea recurvata Lem.) cultivation. Dourados, UFGD, 2009.

\begin{tabular}{|c|c|c|c|c|}
\hline Substratos & $\begin{array}{c}\text { Areia } \\
\left(\mathrm{g} \mathrm{kgsolo}^{-1}\right)\end{array}$ & $\begin{array}{c}\text { Argila } \\
\left(\text { g kgsolo }^{-1}\right)\end{array}$ & $\begin{array}{c}\text { Silte } \\
\left(\text { g kgsolo }^{-1}\right)\end{array}$ & $\begin{array}{c}\text { Dens.Part. } \\
\left(\mathrm{g} \mathrm{cm}^{-3}\right)\end{array}$ \\
\hline LVD & 91,44 & 750,47 & 158,09 & 3,17 \\
\hline AG & 987,34 & 3,59 & 9,07 & 3,03 \\
\hline \multirow[t]{2}{*}{$1 / 3 \mathrm{LVD}+1 / 3 \mathrm{AG}+1 /{ }_{3} \mathrm{MC}$} & 628,67 & 313,33 & 58,00 & 2,30 \\
\hline & $\begin{array}{c}\mathrm{pH} \\
\left(\mathrm{H}_{2} \mathrm{O}\right)\end{array}$ & $\begin{array}{c}\mathrm{P} \\
\left(\mathrm{mg} \mathrm{dm}^{3}\right)\end{array}$ & $\begin{array}{c}\mathrm{K} \\
\left(\mathrm{mmol}_{\mathrm{c}} \mathrm{dm}^{3}\right)\end{array}$ & $\underset{\left(\mathrm{mmol}_{\mathrm{c}} \mathrm{dm}^{3}\right)}{\mathrm{Al}}$ \\
\hline LVD & 4,91 & 0,50 & 0,50 & 3,6 \\
\hline $\mathrm{AG}$ & 7,14 & 3,89 & 1,20 & 0,0 \\
\hline \multirow[t]{2}{*}{$1 / 3 \mathrm{LVD}+1 / 3 \mathrm{AG}+1 /{ }_{3} \mathrm{MC}$} & 6,72 & 39,77 & 0,90 & 0,0 \\
\hline & $\begin{array}{c}\mathrm{Ca} \\
\left(\mathrm{cmol}_{\mathrm{c}} \mathrm{dm}^{3}\right)\end{array}$ & $\begin{array}{c}\mathrm{Mg} \\
\left(\mathrm{cmol}_{\mathrm{c}} \mathrm{dm}^{3}\right)\end{array}$ & $\begin{array}{c}\mathrm{H}+\mathrm{Al} \\
\left(\mathrm{cmol}_{\mathrm{c}} \mathrm{dm}^{3}\right)\end{array}$ & $\mathrm{SB}$ \\
\hline LVD & 0,71 & 0,23 & 2,55 & 9,90 \\
\hline $\mathrm{AG}$ & 1,74 & 0,61 & 0,88 & 24,70 \\
\hline \multirow[t]{2}{*}{$1 / 3 \mathrm{LVD}+1 / 3 \mathrm{AG}+1 /{ }_{3} \mathrm{MC}$} & 1,39 & 0,27 & 1,36 & 17,50 \\
\hline & $\mathrm{T}$ & V\% & & \\
\hline LVD & 35,50 & 28,00 & & \\
\hline $\mathrm{AG}$ & 32,20 & 72,60 & & \\
\hline $1 / 3 \mathrm{LVD}+1 /{ }_{3} \mathrm{AG}+1 /{ }_{3} \mathrm{MC}$ & 31,10 & 56,20 & & \\
\hline
\end{tabular}

$\mathrm{LVD}=$ Latossolo Vermelho Distroférrico; $\mathrm{AG}=$ areia grossa; $\mathrm{MC}=$ moinha de carvão $L V D=$ Oxisol (Typic Haplustox); $A G=$ Thick sand; $M C=$ Charcoal dust

Utilizou-se o delineamento experimental inteiramente casualizados e os tratamentos foram arranjados em esquema fatorial $2 \times 3$ com quatro repetições de 25 sementes.

A moinha de carvão foi obtida pelo peneiramento, em peneira de $5 \mathrm{~mm}$, dos resíduos de carvoaria. A seguir, o material retido na peneira foi lavado com água corrente até remoção total das impurezas e, após secagem à sombra, foi triturado com marreta de $2 \mathrm{~kg}$. Esse material foi novamente peneirado em peneiras de 5 e de $2 \mathrm{~mm}$ sendo utilizado aquele que passou pela peneira de $5 \mathrm{~mm}$ mas ficou retido na peneira de $2 \mathrm{~mm}$ de diâmetro. O latossolo e a areia grossa foram peneirados em peneira de $2 \mathrm{~mm}$.

Os vasos contendo as sementes foram acondicionados em canteiros dentro de viveiro coberto com tela de 50\% de sombreamento, provido de sistema de irrigação por microaspersão descendente, sendo realizadas duas regas diárias que proporcionaram uma lâmina de água equivalente a $2 \mathrm{~mm}$. Quinzenalmente, após o início da emergência, foram feitas contagens do número de plântulas emergidas para o cálculo do índice de velocidade de emergência (IVE) conforme proposto por Maguire (1962).

Aos 180 dias após a semeadura, foi calculada a porcentagem final de emergência $(\% \mathrm{E})$ conforme Popinigis (1985) e as plântulas emergidas foram retiradas dos recipientes e lavadas em água corrente até total remoção do substrato. A seguir, as plântulas foram avaliadas quanto ao diâmetro da porção mais dilatada do caule (com a utilização de um paquímetro digital) e à massa seca total obtida após secagem em estufa com circulação forçada a $65^{\circ} \mathrm{C}$ até peso constante. Os resultados foram submetidos à análise de variância e, havendo diferenças significativas, as médias foram comparadas a 5\% de probabilidade com a utilização do teste F ou do Tukey.

\subsection{Efeito de tratamentos pré-germinativos} na emergência de plântulas de pata de elefante:

Frutos providos de pericarpo e armazenados por 360 dias, nas mesmas condições descritas anteriormente, tive- 
ram o pericarpo removido manualmente e receberam os seguintes tratamentos pré-germinativos: imersão em água natural por $12 ; 24 ; 36 ; 48 ; 72$ e 96 horas (com troca de água a cada 12 horas) e imersão em bioestimulante Stimulate $^{\circledR}$ por 5 minutos nos volumes de $40 ; 80$ e $120 \mathrm{~mL}$ $\mathrm{kg}^{-1}$ de sementes. $\mathrm{O}$ tratamento controle constituiu-se de sementes semeadas imediatamente após a remoção do pericarpo.

Utilizou-se o delineamento experimental inteiramente casualizado com quatro repetições de 25 sementes. A semeadura foi realizada em vasos de polietileno branco de $50 \mathrm{~mL}$, preenchidos com areia grossa, peneirada em peneira de $2 \mathrm{~mm}$. Cada vaso recebeu uma semente colocada a um centímetro de profundidade e coberta posteriormente com areia peneirada. Após a semeadura, os vasos foram acondicionados em viveiro com tela de $50 \%$ de sombreamento, provido de sistema de irrigação por microaspersão descendente, onde permaneceram por 60 dias, recebendo uma lâmina de água diária equivalente a $2 \mathrm{~mm}$.

Após o início da emergência, foram feitas contagens do número de plântulas emergidas para o cálculo do índice de velocidade de emergência (IVE) conforme proposto por Maguire (1962) e, ao final do período experimental, foi calculada a porcentagem final de emergência $(\% \mathrm{E})$ conforme Popinigis (1985). Os resultados foram submetidos à análise de variância e as médias foram comparadas por teste de Scott-Knott até 5\% de probabilidade.

\section{RESULTADOS E DISCUSSÃO}

Os dados de índice de velocidade de emergência (IVE), porcentagem de emergência $(\% \mathrm{E})$, diâmetro do caule (DC) e massa seca (MS) das plântulas de Beaucarnea recurvada Lem. foram submetidos à análise de variância e suas significâncias e médias gerais, em função dos fatores estudados, são apresentados na Tabela 2 .

Tabela 2. Resumo das análises de variância do índice de velocidade de emergência (IVE), porcentagem de emergência (\%E), diâmetro do caule (DC) e matéria seca total (MS) de plântulas de pata de elefante (Beaucarnea recurvata Lem.) Dourados, UFGD, 2009.

Table 2. Variance Analyses of emergence speed index (IVE), emergence percentage (\%E), stem diameter (DC) and total dry matter (MS) of ponytail seedlings (Beaucarnea recurvata Lem.) in function of the combined effects of pericarp presence and substrata utilized. Dourados, UFGD, 2009.

\begin{tabular}{|c|c|c|c|c|c|}
\hline & & \multicolumn{4}{|c|}{ Quadrados médios } \\
\hline FV & GL & IVE & \%E & DC & MS \\
\hline Sementes & 1 & $0,67^{* *}$ & $3174,00^{* *}$ & $0,003^{\text {ns }}$ & $0,001^{\text {ns }}$ \\
\hline Substratos & 2 & $0,15^{*}$ & $558,00^{*}$ & $0,070^{* *}$ & $0,196^{* *}$ \\
\hline Sementes x Substratos & 2 & $0,06^{\text {ns }}$ & $122,00^{\text {ns }}$ & $0,004^{\text {ns }}$ & $0,006^{\text {ns }}$ \\
\hline Resíduo & 18 & 0,02 & 141,55 & 0,001 & 0,008 \\
\hline CV(\%) & & 21,47 & 24,53 & 4,48 & 10,56 \\
\hline Média geral & & 0,78 & 48,50 & $0,97 \mathrm{~mm}$ & 0,87 \\
\hline
\end{tabular}

** significativo, a $1 \%$ de probabilidade, pelo teste $\mathrm{F}$; * significativo, a 5\% de probabilidade, pelo teste $\mathrm{F}$; ${ }^{\text {ns }}$ não significativo

** Significant at $1 \%$ probability by $\mathrm{F}$ test; * Significant at $5 \%$ probability by $\mathrm{F}$ test; ${ }^{\text {ns }}$ not significant

Houve efeito isolado da presença do pericarpo $(\mathrm{p}<0,01)$ somente sobre o IVE e a \%E (Tabela 2). Para estas variáveis, os maiores valores foram observados na ausência do pericarpo (Tabela 3). Para o diâmetro do caule e massa seca da planta não foram observadas diferenças estatísticas ( $>00,05)$ (Tabela 2).

A remoção do pericarpo das sementes de pata-de-elefante propiciou maior $\% \mathrm{E}$ em menor tempo. Como não foram observadas diferenças estatísticas em relação ao diâmetro do caule e massa fresca das plantas, a prática da remoção do pericarpo pode ser indicada para a cultura como forma de otimização da produção de mudas (Tabela 3).

Os resultados observados para o IVE e \%E deste trabalho estão de acordo com a literatura, uma vez que para a produção de mudas de algumas espécies, recomenda-se a remoção do pericarpo das sementes, pois este processo acelera e uniformiza o processo germinativo (YOCUM, 1964 citado por FERREIRA e GENTIL, 2006).

Segundo Perez (2004), a ocorrência de germinação, quando a casca ou o pericarpo é removido, denota a existência de uma barreira mecânica para o embrião. Dessa maneira, os resultados obtidos no presente trabalho podem sugerir a presença de alguma substância inibidora no pericarpo de sementes de pata-de-elefante, entretanto, é necessário que novos estudos sejam realizados para determinar se a espécie em questão apresenta algum tipo de dormência.

Os maiores resultados de IVE, DC, \%E e MS foram observados no substrato constituído por $1 /{ }_{3} \mathrm{LVD}+1 /{ }_{3} \mathrm{AG}+$ ${ }_{3}^{1} \mathrm{MC}$, sendo que para as duas últimas variáveis, os valores foram estatisticamente iguais $(\mathrm{p}<0,05)$ àqueles obtidos no substrato constituído por AG (Tabela 3).

Estes resultados podem ser atribuídos aos menores valores de densidade (Tabela 1) observados no substrato constituído por $1 /{ }_{3} \mathrm{LVD}+1 /{ }_{3} \mathrm{AG}+1 /{ }_{3} \mathrm{MC}$. A moinha de carvão tem uma estrutura altamente porosa que, se misturada ao solo ou substrato, pode aumentar sua porosidade e a capacidade de retenção de água, podendo, dessa maneira, facilitar a penetração das radículas quando comparado a outros materiais (ZANETTI et al., 2003), favorecendo, dessa forma, a emergência de plântulas de pata-de-elefante quando comparada aos demais substratos utilizados. 
Tabela 3. Índice de velocidade de emergência (IVE), porcentagem de emergência (\%E), diâmetro do caule (DC) e matéria seca total (MS) de plântulas de pata de elefante (Beaucarnea recurvata Lem.) em função dos efeitos isolados da presença do pericarpo e dos substratos utilizados. Dourados, UFGD, 2009.

Table 3. Emergence speed index (IVE), emergence percentage (\%E), stem diameter (DC) and total dry matter (MS) of ponytail seedlings (Beaucarnea recurvata Lem.) in function of the combined effects of pericarp presence and substrata utilized. Dourados, UFGD, 2009.

\begin{tabular}{|c|c|c|c|c|}
\hline Sementes & IVE & $\mathbf{\% E}$ & $\mathbf{D C}$ & MS \\
\hline Sem pericarpo & $0,95 \mathrm{a}$ & $60,00 \mathrm{a}$ & $0,96 \mathrm{a}$ & $0,86 \mathrm{a}$ \\
\hline Com pericarpo & $0,61 \mathrm{~b}$ & $37,00 \mathrm{~b}$ & $0,99 \mathrm{a}$ & $0,87 \mathrm{a}$ \\
\hline Substratos & IVE & $\% \mathrm{E}$ & $\mathrm{DC}$ & $\mathrm{MS}$ \\
\hline $\mathrm{AG}$ & $0,70 \mathrm{~b}$ & $47,00 \mathrm{ab}$ & $0,93 \mathrm{~b}$ & $0,96 \mathrm{a}$ \\
\hline $\mathrm{LVD}$ & $0,10 \mathrm{~b}$ & $41,00 \mathrm{~b}$ & $0,91 \mathrm{~b}$ & $0,69 \mathrm{~b}$ \\
\hline $1 / 3 \mathrm{LVD}+1 /{ }_{3} \mathrm{AG}++1 / 3 \mathrm{MC}$ & $0,94 \mathrm{a}$ & $57,50 \mathrm{a}$ & $1,09 \mathrm{a}$ & $0,95 \mathrm{a}$ \\
\hline $\mathrm{CV}(\%)$ & 21,47 & 24,53 & 4,48 & 10,56 \\
\hline Média geral & 0,78 & $48,50 \%$ & $0,97 \mathrm{~mm}$ & $0,87 \mathrm{~g}$ \\
\hline
\end{tabular}

Médias seguidas de mesma letra na coluna não diferem entre si (F 1\% para sementes; Tukey 5\% para substratos) $\mathrm{AG}=$ areia grossa; $\mathrm{LVD}=\mathrm{Latossolo}$ Vermelho Distroférrico; $\mathrm{MC}=$ moinha de carvão.

Medium followed by the same letter, in the column, do not differ among themselves ( $F$ 1\% for seeds; Tukey $5 \%$ for substrata) AG= Thick sand;LVD= Oxisol (Typic Haplustox); $M C=$ Charcoal dust

Os valores dos atributos físicos do substrato constituído por $1 /{ }_{3} \mathrm{LVD}+{ }_{3} \mathrm{AG}+{ }_{3} / \mathrm{MC}$ associados aos seus atributos químicos (Tabela 1) podem ter proporcionado às plântulas de pata-de-elefante os maiores diâmetros de caule e quantidades de matéria seca total (Tabela 3), que são características vegetais dependentes do ambiente de crescimento encontrado nos substratos.
A tabela 4 apresenta os valores de \%E e IVE obtidos em função dos diferentes tratamentos pré-germinativos propostos.

Não houve efeito significativo dos tratamentos pré-germinativos para a $\% \mathrm{E}$, que apresentou valor médio de 80,40 (Tabela 4). Já para o IVE, a imersão das sementes em água por $96 \mathrm{~h}$ proporcionou valor significativamente maior $(\mathrm{p}<0,05)$ a todos os demais tratamentos propostos (Tabela 4).

Tabela 4. Porcentagem de emergência (\%E) e índice de velocidade de emergência (IVE) de pata de elefante (Beaucarnea recurvata Lem.) em função do tratamento pré-germinativo. Dourados, UFGD, 2009.

Table 4. Emergence percentage (\%E) and emergence speed index (IVE) of ponytail Beaucarnea recurvata Lem.) in function of pregerminative treatment. Dourados, UFGD, 2009.

\begin{tabular}{|c|c|c|}
\hline Tratamentos & $\mathbf{\% E}$ & IVE \\
\hline controle & $78,75 \mathrm{a}$ & $1,25 \mathrm{~b}$ \\
\hline Imersão em água por $12 \mathrm{~h}$ & $80,00 \mathrm{a}$ & $1,00 \mathrm{~b}$ \\
\hline Imersão em água por $24 \mathrm{~h}$ & $78,75 \mathrm{a}$ & $1,00 \mathrm{~b}$ \\
\hline Imersão em água por $36 \mathrm{~h}$ & $84,00 \mathrm{a}$ & $1,00 \mathrm{~b}$ \\
\hline Imersão em água por $48 \mathrm{~h}$ & $85,25 \mathrm{a}$ & $1,00 \mathrm{~b}$ \\
\hline Imersão em água por $72 \mathrm{~h}$ & $78,75 \mathrm{a}$ & $1,00 \mathrm{~b}$ \\
\hline Imersão em água por $96 \mathrm{~h}$ & $89,25 \mathrm{a}$ & $1,75 \mathrm{a}$ \\
\hline Bioestimulante $40 \mathrm{~mL} \mathrm{~kg}^{-1}$ & $68,00 \mathrm{a}$ & $0,75 \mathrm{~b}$ \\
\hline Bioestimulante $80 \mathrm{~mL} \mathrm{~kg}^{-1}$ & $80,00 \mathrm{a}$ & $1,00 \mathrm{~b}$ \\
\hline Bioestimulante $120 \mathrm{~mL} \mathrm{~kg}^{-1}$ & $81,25 \mathrm{a}$ & $1,00 \mathrm{~b}$ \\
\hline $\mathrm{CV}(\%)$ & 11,63 & 25,48 \\
\hline Média geral & $80,40^{\text {ns }}$ & $1,07 * *$ \\
\hline
\end{tabular}

Médias seguidas de mesma letra, na coluna, não diferem entre si (Scott-Knott 5\%)

** tratamentos significativos, a 1\% de probabilidade, pelo teste $\mathrm{F}$; ${ }^{\text {ns }}$ tratamentos não significativos, a $5 \%$ de probabilidade, pelo teste $\mathrm{F}$

Medium followed by the same letter, in the column, do not differ among themselves (Scott-Knott 5\%).

** Significant at $1 \%$ probability by $\mathrm{F}$ test; ${ }^{\mathrm{ns}}$ not significant at $5 \%$ probability by $\mathrm{F}$ test 
O efeito benéfico da imersão em água também foi observado em sementes de tucumã (Astrcaryum aculeatum Meyer) por Ferreira e Gentil( 2006), e em sementes de tarumã (Vítex megapotamica (Spreng.) Moldenke) por Vianna e Koehler (2007), uma vez que a pré-embebição das sementes em água, sob temperatura ambiente, pode aumentar a velocidade de germinação das sementes, pois acelera a absorção de água e ativação dos processos enzimáticos responsáveis pela germinação (WILLAN, 1990).

Embora Garcia et al (2006) tenham observado efeitos benéficos da utilização do Bioestimulante sobre a porcentagem de germinação de sementes Strelitzia reginae, o mesmo não foi observado para Beaucarnea recurvata .

\section{CONCLUSÕES}

Nas condições em que o trabalho foi realizado, recomenda-se a remoção do pericarpo das sementes de $B$. recurvata e a utilização de substrato composto por Latossolo Vermelho Distroférico + areia grossa + moinha de carvão, na proporção de 1:1:1 em volume.

\section{AGRADECIMENTOS}

À Fundação de Desenvolvimento Científico e Tecnológico do Mato Grosso do Sul (FUNDECT), pelo apoio financeiro, e à CAPES, pela concessão de bolsa de iniciação científica.

\section{REFERÊNCIAS}

CARVALHO, N. M.; NAKAGAWA, J. Sementes: ciência, tecnologia e produção. 4. ed. Jaboticabal: Funep, 2000. 588p.

EMBRAPA- Empresa Brasileira de Pesquisa Agropecuária. Manual de métodos de análise de solo. 2.ed. Rio de Janeiro: Embrapa/CNPS, 1997. 212p. (Documento,1).

FERREIRA, S. A. N., GENTIL, D. F. O. Extração, embebição e germinação de sementes de tucumã (Astrocaryum acueatum). Acta Amazonica, Manaus, v. 36, n. 2, p.141146, 2006.

FIGLIOLIA, M. B.; OLIVEIRA, E. C.; PIÑA-RODRIGUES, F. C. M. Análise de Sementes. In: AGUIAR, I. B.; PIÑA-RODRIGUES, F. C. M.; FIGLIOLIA, M. B. (Ed.). Sementes Florestais Tropicais. Brasília: ABRATES, p. 37-74, 1993.

GARCIA, A. S.; BRANQUINHO, E. G. A.; MENUCHI, A. C. T. P.; ERLACHER, K. C.; DOMINGUES, M. C. S. Efeito de reguladores vegetais na germinação e desenvolvimento da sementes Strelitzia reginae. Thesis, São Paulo, ano III, v. 5, p. 161-176, 2006.

GROLLI, P. R. Composto de lixo domiciliar como condicionador de substrato para plantas arbóreas. Porto Alegre: Universidade Federal do Rio Grande do Sul, 1991. 125p. Dissertação (Mestrado em Fitotecnia).
LORENZI, H.; SOUZA, H. M. Plantas ornamentais no Brasil: arbustivas, herbáceas e trepadeiras. Nova Odessa, SP: Plantarum, 1999, 1088p.

KÖPENN W. Climatologia: com um Estudiu de los Climas de la Tierra. México: Fondo de Cultura Económica, 1948, 478p.

MAGUIRE, I. D. Speed of germination aid in selection and evaluation for seedling emergence and vigor. Crop Science, Madson, n. 1, p. 176-177, 1962.

MARQUES, R. W. C.; CAIXETA FILHO, J. V. Avaliação da sazonalidade do mercado de flores e plantas ornamentais no Estado de São Paulo. Revista Brasileira de Horticultura Ornamental, Campinas, v. 9, n. 2, p. 143-160, 2003.

PEREIRA, L. V.; ANDRADE, R. P.; KARIA, C. T. Efeitos do pericarpo e do tratamento e sementes no estabelecimento de Arachis pintoi. In: REUNIÃO ANUAL DA SOCIEDADE BRASILEIRA DE ZOOTECNIA, 33, Fortaleza, 1996. Anais. Fortaleza: SBZ, p. 392-394, 1996.

PERES, S.C.J.G.A. Envoltórios. In: FERREIRA, A.G.; BORGUETTI. (orgs.). Germinação: do básico ao aplicado. Porto Alegre: Artmed, p.125-135, 2004.

POPINIGIS, F. Fisiologia de sementes. Brasília: Agiplan, 1985, 289p.

ROSSETTO, C. A. V.; ALVES, E. P. Tratamentos pré-germinativos em sementes de Arachis pintoi. Ciência e Agrotecnologia, Lavras, v.32, n.1, p.147-179, 2008.

TANIO, D. S.; SIMÕES, S. C. Cadeia de suprimentos de flores e plantas ornamentais no Brasil - uma nova abordagem para aumentar a participação do setor no mercado internacional. Estudos realizados - GELOG - UFSC, 2005.

TERCEIRO NETO, C. P. C.; HERNANDEZ, F. F. F., BEZERRA, F. C., SOUSA, R. F., CAVALCANTI, M. L. F. Efeito da concentração salina da solução nutritiva na aclimatização de plantas micropropagadas de Violeta Africana (Saintpaulia ionantha Wendl). Revista de biologia e ciência do solo, João Pessoa, v.4, n.2, p., 2004.

VARELA, V. P.; COSTA, S. S.; RAMOS, M. B. P. Influência da temperatura e do substrato na germinação de sementes de itaubarana (Acosmium nitens (Vog.) Yakovlev - Leguminosae, Caesalpinoideae. Acta Amazônica, Manaus, v.35, n.1, p.35-39, 2005.

VIANNA, E.; KOEHLER, A.B. Tratamentos simplificados para germinação de sementes de tarumã (Vitex megapotamica (Spreng.) Moldenke). Revista Acadêmica, Curitiba, v. 5, n. 2, p.189-193, 2007. 
VIEIRA, E. L., CASTRO, P. R. C. Ação de stimulate no desenvolvimento inicial de plantas de algodoeiro (Gossypium hirsutum L.). Piracicaba: USP. Dept. Ciências Biológicas, 2002. 3p.

ZANETTI, M.; CAZETTA, J. O.; MATTOS JÚNIOR, D.; CARVALHO, S. A. de. Uso de subprodutos de carvão vegetal na formação do porta-enxerto limoeiro 'cravo' em ambiente protegido. Revista Brasileira de Fruticultura, Jaboticabal, v. 25, n. 3, p.508-512, 2003.

WILLAN, R. L. Seed pretreatment. Humleaback, Danida Forest Seed Centre, 1990. 19p. (Lecture note, c-10). 